\title{
MATLAB Simulation of Subspace based High Resolution Direction of Arrival Estimation Algorithm
}

\author{
Pallavi Agrawal \\ Department of ECE, \\ Maulana Azad \\ National Institute of Technology, \\ Bhopal (M. P.)-462051
}

\author{
Madhu Shandilya \\ Department of ECE, \\ Maulana Azad \\ National Institute of Technology, \\ Bhopal (M. P.)-462051
}

\begin{abstract}
Over the past decades there has been technological breakthrough from analog to digital. Ever since the world war and also the invention of electromagnetic radio, there has always been an urge to know from which direction the signals were arriving to the listener's direction finding receiver. The methodology of determining the direction of arrival (DOA) of incoming signals impinging on the uniform antenna array has drastically changed from conventional methods to subspace based methods. The main goal of this paper is to provide a comparative study of various classical multi-channel DOA algorithms and subspace based high resolution multi-channel DOA algorithms for multiple signals which are arriving at different DOA. Also detailed algorithm analysis and performance evaluation for subspace based multiple signal classification (MUSIC) algorithm is provided. It is shown that subspace based high resolution multi-channel MUSIC algorithm is statistically efficient and is a promising method for use against military communication in an electronic warfare direction finding system.
\end{abstract}

\section{Keywords}

Direction of Arrival, Subspace, Electromagnetic radio, MUSIC.

\section{INTRODUCTION}

Direction finding (DF) systems uses multiple antenna arrays arranged in a pattern along with one or many receivers at its back end to estimate the direction of arrival (DOA) of incoming signal. For this, it uses the characteristics of signal impinging on each of the elements of the antenna array such as signals amplitude, phase or time of arrival. DF systems have wide applications such as wireless communication, tracking of various objects, radars, astronomy, electronic warfare and signal intelligence [1], [2]. While estimating DOA, multipath fading environment and cross interference becomes a bottleneck [3-5]. To mitigate these problems, smart antennas are employed with varying weights instead of fixed antennas which provide efficient algorithms for DOA estimation [6-8]. Here both classical multichannel DOA algorithms and subspace based high resolution DOA algorithms are compared. Also performance evaluation of MUSIC algorithm [9-12] is provided in detail.

This paper is organized as follows. Section II describes system model and the basics of DOA estimation. Section III discusses the basic mathematical development of classical estimation methods such as Delay and Sum beam-former [13] and Capons Minimum Variance Distortion-less Response (MVDR) beam-former [14], [15] and high resolution MUSIC algorithm. Section IV provides the performance evaluation and simulation results of classical and subspace based methods. Section V provides the detailed analysis of subspace based high resolution MUSIC algorithm. This is done by varying the number of array elements, array spacing, number of snapshots, signal to noise ratio of incoming signals and incident angle difference. Section VI summarizes the paper with brief conclusion followed by references used. All the simulations are done using MATLAB toolbox [16].

During the course of paper the following assumptions are made. Signal source is a point source. Narrowband

assumption is made so that all the array elements in an antenna array receive the incoming signal. It is also assumed that it is white Gaussian, identically and independent noise source with mean 0 and variance $\sigma^{2}$.

\section{System Model}

Consider a general beamforming system as shown in the Fig. 1. It consists of Uniform Linear Array consisting of $0,1,2$ $\mathrm{N}-1$ antenna elements, each having a spacing of half wavelength between the elements.

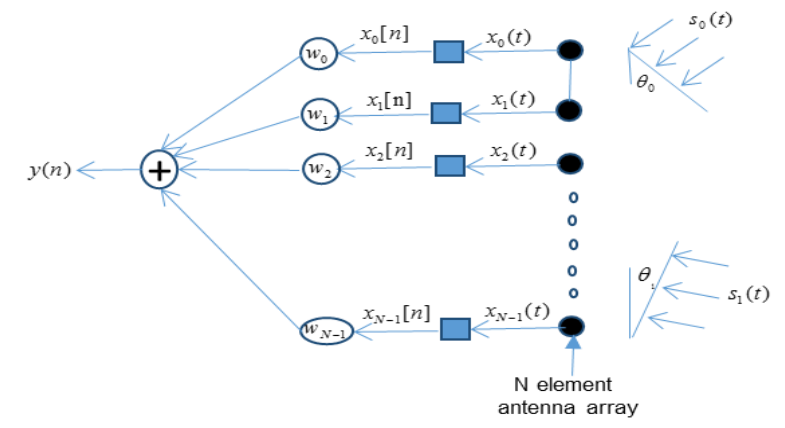

Fig. 1: Block Diagram of General Beamforming system

Multiple signals $s_{0}(t), s_{1}(t)$ etc. impinge on antenna elements makes an angle $\theta_{0}, \theta_{1}$ respectively. Output of each antenna from $\mathrm{N}$ element array is weighted by a complex number and simulated to give a single output $\mathrm{y}(\mathrm{n})$. Thus a steering vector $a(\varphi)$ is made which is vector of individual weights. This method of steering vector generation characterises any beamforming system. It is assumed that the phase of the baseband signal received at the element 0 is zero. Also the phase of the other elements will be measured with respect to this received at element zero.

Using the narrowband assumption, the signal received by the $\mathrm{k}^{\text {th }}$ element at any time $\mathrm{t}$ is given by-

$$
x_{k}(n T) \approx s(n T) e^{-j 2 \pi f_{c} \Delta t_{k}} \approx s(n T) e^{\frac{-j 2 \pi n d \sin \theta}{\lambda}}
$$

Where $c=\lambda f_{c}$, 'c' is the speed of light, $\mathrm{f}_{\mathrm{c}}$ is the carrier frequency, $\mathrm{T}$ is the symbol period, $\mathrm{s}(\mathrm{t})$ is the baseband signal, $\mathrm{x}_{\mathrm{k}}(\mathrm{t})$ is the signal received by the $\mathrm{k}^{\text {th }}$ element, 
$\Delta t_{k}=\frac{n d \sin \theta}{c}, \mathrm{n}$ is the time index and ' $\lambda$ ' is the wavelength of the propagating wave.

Equation (1) can also be written as-

$$
x_{k}(n T) \approx s(n T) e^{\frac{-j \pi k \sin \theta}{\lambda}}
$$

In discrete notation it can be written as,

$$
x_{k}[n] \approx s[n] e^{\frac{-j \pi k \sin \theta}{\lambda}}=s[n] a_{k}(\theta)
$$

For $\mathrm{m}$ incoming signals, the $\mathrm{n}^{\text {th }}$ symbol of $\mathrm{i}^{\text {th }}$ signal denoted by $s_{i}[n]$, the sampled signals can be expressed as:

$$
x_{k}[n]=\sum s_{i}[n] a\left(\theta_{i}\right)
$$

Considering all the array elements, equation (4) can be written in matrix notation as:

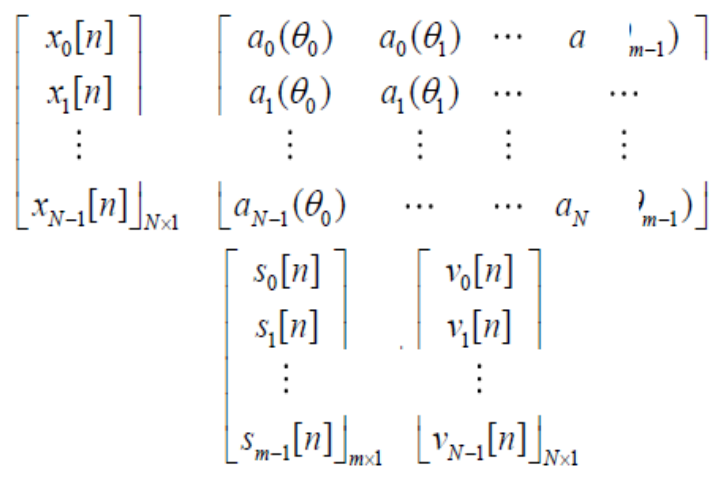

Where $\mathrm{v}[\mathrm{n}]$ is the noise vector.

Equation (5) can be written in compact matrix notation as:

$$
\begin{aligned}
x_{n} & =\left[\begin{array}{llll}
\mathbf{a}\left(\theta_{0}\right) & \mathbf{a}\left(\theta_{1}\right) & \cdots & \mathbf{a}\left(\theta_{m-1}\right)
\end{array}\right] \mathbf{s}_{n}+\mathbf{v}_{n} \\
& =\mathbf{A} \mathbf{s}_{n}+\mathbf{v}_{n}
\end{aligned}
$$

The columns of matrix $\mathbf{A}$ are called the steering vectors of signal. Thus array output can be computed by

$$
y[n]=\sum_{k=0}^{N-1} w_{k} x_{k}[n]=\mathbf{w}^{\mathbf{H}} \mathbf{x}_{n}=s_{0}[k] \mathbf{w}^{\mathbf{H}} \mathbf{a}\left(\theta_{0}\right)
$$

If there are two digitally modulated signals present, $\mathrm{s}_{0}(\mathrm{t})$ and $\mathrm{s}_{1}(\mathrm{t})$ with angles of arrival $\theta_{0}$ and $\theta_{1}$, the beam-former output is given by

$$
y[k]=s_{0}[k] \mathbf{w}^{\mathbf{H}} \mathbf{a}\left(\theta_{0}\right)+s_{1}[k] \mathbf{w}^{\mathbf{H}} \mathbf{a}\left(\theta_{1}\right)
$$

So the main objective is to find the best algorithm which is able to identify these multiple angles of arrival with high resolution and also once algorithm is identified, to carry out the detailed study of it by varying its enabling parameters.

\section{Mathematical Description of Various methods to estimate DOA}

\subsection{Delay and Sum Beamformer}

In Delay and Sum beam-former, the antenna array beam is steered in one direction at a time and output signal power is computed. The location which gives maximum power is the DOA estimate. All the weights are given magnitude of $1 / \mathrm{N}$ but each having different phase.

$$
w=\frac{1}{N} a(\theta)=\frac{1}{N}\left[\begin{array}{lllll}
1 & e^{-j \omega} & e^{-j 2 \omega} & \ldots & e^{-j(N-1) \omega}
\end{array}\right]^{T}
$$

Thus beam-former output is given by:

$$
y[n]=\mathbf{w}^{H} \mathbf{x}_{n}
$$

Power is given by:

$$
\begin{aligned}
P(\theta) & =E\left[y^{H} y\right]=E\left|\mathbf{w}^{H} \mathbf{x}_{n}\right|^{2} \\
& =E\left|\mathbf{a}(\theta)^{H} \mathbf{x}_{n}\right|^{2}=\mathbf{a}(\theta)^{H} \mathbf{R}_{x x} \mathbf{a}(\theta)
\end{aligned}
$$

Here $\mathbf{R}_{x x}$ is the spatial Covariance Matrix $=E\left[\mathbf{x}_{n} \mathbf{x}_{n}^{H}\right]$. This method uses all the degrees of freedom and adjusts the weight vector in the direction where maximum power is obtained. As such it is a very simple method but in order to increase the DOA resolution, number of antenna elements in the array had to be increased.

\subsection{Capons Minimum Variance Distortion- less Response (MVDR) Beam-former}

In order to resolve sources placed closer, Capon proposed an improved method of DOA estimation. It is an optimization problem in which for each measured angle $\theta$, signal power is minimized with respect to $\mathbf{W}$, with an additional constraint that $\mathbf{W}^{H} \mathbf{a}(\theta)=1$.Mathematically,

$\min _{w} E[|y(k)|]^{2}=\min _{w} \mathbf{w}^{H} \mathbf{R}_{x x} \mathbf{w}$. It is also called as Minimum Variance Distortionless Response (MVDR) beamformer filter which minimizes the power due to noise or any other unwanted signals coming from undesired direction while maintaining gain as fixed in the look direction $\theta$. The optimal weight of MVDR is given by-

$$
w_{M V D R}=\frac{R_{x x}^{-1} \mathbf{a}(\theta)}{\mathbf{a}^{H}(\theta) R_{x x}^{-1} \mathbf{a}(\theta)}
$$

Using this weight vector, the spatial spectrum can be calculated using the below equation as-

$$
\mathbf{P}_{C A P}(\theta)=\frac{1}{\mathbf{a}^{H}(\theta) \mathbf{R}_{x x}^{-1} \mathbf{a}(\theta)}
$$

Where $\mathbf{a}(\theta)$ is the steering vector for the desired direction and $\mathbf{W}$ is the vector of complex weights. This method gives a significant improvement over the previous method as it minimizes power from undesired directions. It has disadvantage that it requires inverse matrix computation and may sometimes give misleading results if highly correlated signals are present. 


\subsection{Subspace based Multiple Signal Classification (MUSIC) Beamformer}

These are high performance DF algorithms having the capability to determine DOA of multiple signals with resolution better than one degree. The block diagram of MUSIC algorithm is shown in Fig. 2.

These are high performance DF algorithms having the capability to determine DOA of multiple signals with resolution better than one degree. The block diagram of MUSIC algorithm is shown in Fig. 2.

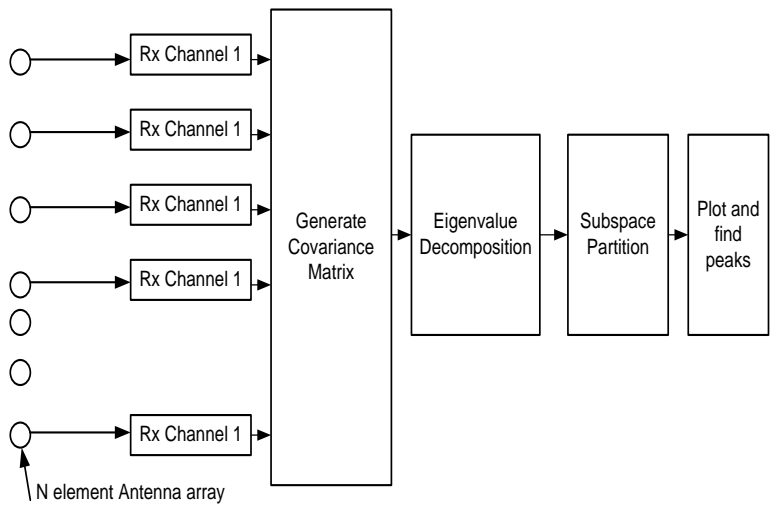

Fig. 2: Block Diagram of MUSIC Algorithm

In order to estimate DOA, search all the steering vectors and find those which are orthogonal to the noise subspace. From equation (6), it is known that $\mathbf{x}_{n}=\mathbf{A} \mathbf{s}_{n}+\mathbf{v}_{n}$. In absence of noise, signal received by each element of array is given by $\mathbf{x}_{n}=\mathbf{A} \mathbf{s}_{n}$. These elements of data vector $\mathbf{x}_{n}$ span the signal subspace. The spatial covariance matrix $\mathbf{R}_{x x}$ can be written as:

$$
\begin{aligned}
\mathbf{R}_{x x} & =E\left[\begin{array}{ll}
\mathbf{x}_{n} & \mathbf{x}_{n}^{H}
\end{array}\right]=E\left[\begin{array}{ll}
\left(\mathbf{A} s_{n}+\mathbf{v}_{n}\right) & \left(\mathbf{A} s_{n}+\mathbf{v}_{n}\right)^{H}
\end{array}\right] \\
& =\mathbf{A} E\left[\begin{array}{ll}
\mathbf{S}_{n} & \mathbf{s}_{n}^{H}
\end{array}\right] \mathbf{A}^{H}+E\left[\begin{array}{ll}
\mathbf{v}_{n} & \mathbf{v}_{n}^{H}
\end{array}\right] \\
& =\mathbf{A} \mathbf{R}_{s s} \mathbf{A}^{H}+\sigma^{2} \mathbf{I}_{N \times N}
\end{aligned}
$$

Where $\mathbf{R}_{s s}=E\left[\begin{array}{ll}S_{n} & s_{n}^{H}\end{array}\right]$.

Any vector $\mathbf{q}_{n}$ which is orthogonal to columns of $\mathbf{A}$ can be written as-

$$
\mathbf{R}_{x x} \mathbf{q}_{n}=\left(\mathbf{A} \mathbf{R}_{s s} \mathbf{A}^{H}+\sigma^{2} \mathbf{I}\right) \mathbf{q}_{n}=0+\sigma^{2} \mathbf{I} \mathbf{q}_{n}=\sigma^{2} \mathbf{q}_{n}
$$

Thus for Eigen vector $\mathbf{q}_{n}$, Eigen value is $\sigma^{2}$. A has dimensions of $N \times m$, thus there will be $N-m$ linearly independent Eigen vectors whose Eigen values will be $\sigma^{2}$. Space spanned by $N-m$ Eigen vectors is called the noise subspace. If $\mathbf{q}_{s}$ is the Eigen vector of $\mathbf{A} \mathbf{R}_{s s} \mathbf{A}$ then-

$$
\begin{aligned}
\mathbf{R}_{x x} \mathbf{q}_{s} & =\left(\mathbf{A R}_{s s} \mathbf{A}^{H}+\sigma^{2} \mathbf{I}\right) \mathbf{q}_{s}=\sigma_{s}^{2} \mathbf{q}_{s}+\sigma^{2} \mathbf{I} \mathbf{q}_{s} \\
& =\left(\sigma_{s}^{2}+\sigma^{2}\right) \mathbf{q}_{s}
\end{aligned}
$$

Here $\mathbf{q}_{s}$ is also Eigen vector of $\mathbf{R}_{x x}$ with Eigen value $\left(\sigma_{s}^{2}+\sigma^{2}\right), \quad \sigma_{s}^{2}$ is the Eigen value of $\mathbf{A} \mathbf{R}_{s s} \mathbf{A}^{H}$. Space spanned by these $m$ vectors is the signal subspace. Thus the Eigen decomposition of $\mathbf{R}_{x x}$ can be expressed as-

$$
\mathbf{R}_{x x}=\mathbf{Q D Q}^{H}=\left[\begin{array}{ll}
\mathbf{Q}_{s} & \mathbf{Q}_{n}
\end{array}\right]\left[\begin{array}{cc}
\mathbf{D}_{s} & 0 \\
0 & \sigma^{2} \mathbf{I}
\end{array}\right]\left[\begin{array}{ll}
\mathbf{Q}_{s} & \mathbf{Q}_{n}
\end{array}\right]^{H}
$$

Where $\mathbf{Q}_{s}$ is a $N \times m$ matrix whose columns are signal Eigen vectors which corresponds to signal subspace. $\mathbf{Q}_{n}$ is an $N \times(N-m)$ matrix whose columns are noise Eigen vectors. $\mathbf{D}$ is an $r \times r$ diagonal matrix whose diagonal elements are "signal" Eigen values and $\mathbf{I}$ is an $N \times N$ identity matrix whose diagonal elements are "noise" Eigen values. For one of the incoming signals if $\mathbf{a}(\theta)$ is the steering vector, then $\mathbf{a}(\theta)^{H} \mathbf{Q}_{n}=0$. When $\theta$ is the DOA of the incoming signal, then MUSIC spectrum will assume a very large value which can be calculated as-

$$
\mathbf{P}_{M U S I C}(\theta)=\frac{1}{\mathbf{a}^{H}(\theta) \mathbf{Q}_{n} \mathbf{Q}_{n}^{H} \mathbf{a}(\theta)}
$$

Using MUSIC algorithm, first generate the covariance matrix, perform its Eigen decomposition and find the peaks. The associated angles provides the estimates for DOA.

\section{SIMULATION RESULTS}

Here the comparison between simulation results of various Direction of Arrival algorithms has been provided. Fig. 3 shows the simulations for Delay and Sum beam-former, MVDR beam-former and MUSIC algorithm for three incoming signals.

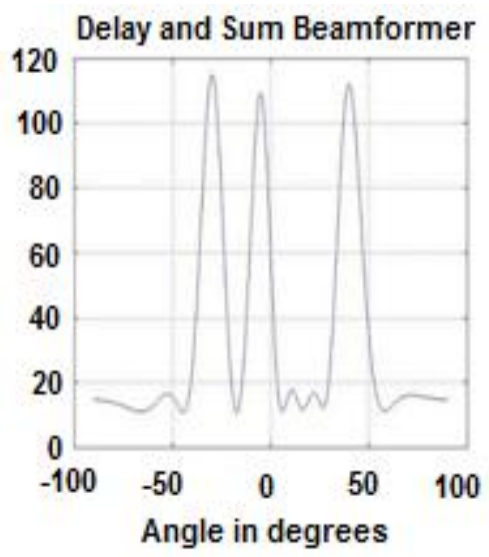

(a) 


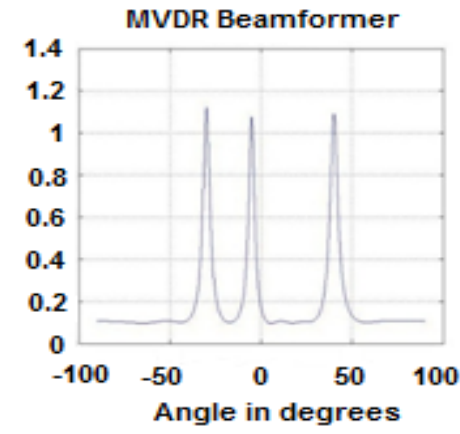

(b)

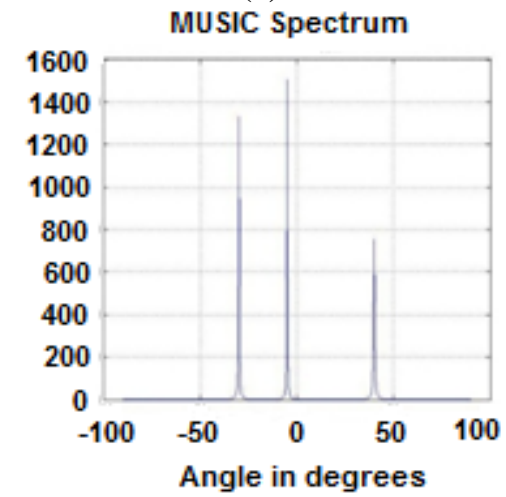

(c)

Fig. 3: MATLAB Simulation Results of various DOA algorithms for (a) Delay and Sum beam-former (b) MVDR beam-former, and (c) MUSIC algorithm

As seen, Delay and Sum beam-former is able to identify the signals but the locations are not represented by sharp peaks due to spectral leakage. MVDR and MUSIC also identifies the three signals, but MUSIC is able to represent the locations with more prominent peaks. Delay and Sum and MVDR also produce spurs at other locations, whereas MUSIC does not produce such spurs. Thus MUSIC algorithm is able to identify multiple signals accurately compared to other DOA algorithms.

\section{SIMULATION RESULTS OF MUSIC ALGORITHM FOR DOA ESTIMATION}

From the above simulations it was seen that MUSIC algorithm was better than the classical and other beam-former method. Further carried out simulations in detail as provided by MUSIC algorithm by varying the number of array elements, array spacing, number of snapshots, signal to noise ratio of incoming signals and incident angle difference. For carrying out simulations considered four signals with angle of incidence as $5^{\circ}, 20^{\circ}, 45^{\circ}, 70^{\circ}$. These signals are uncorrelated. Noise considered is additive white Gaussian noise. Element spacing is half wavelength, the number of array elements considered for simulation is 10 , SNR is $20 \mathrm{~dB}$ and the total number of snapshots considered is 200 .

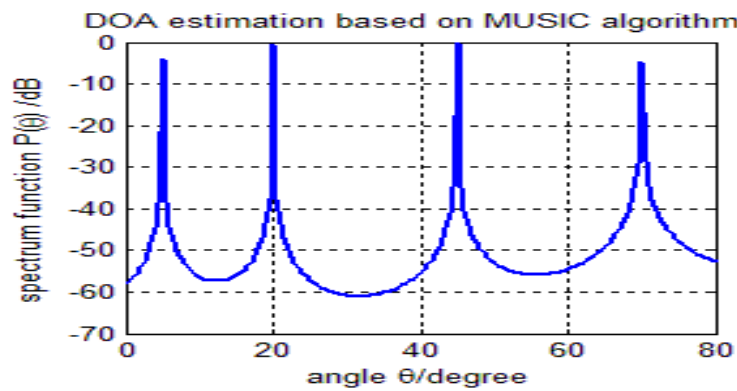

(a)

DOA estimation based on MUSIC algorithm by

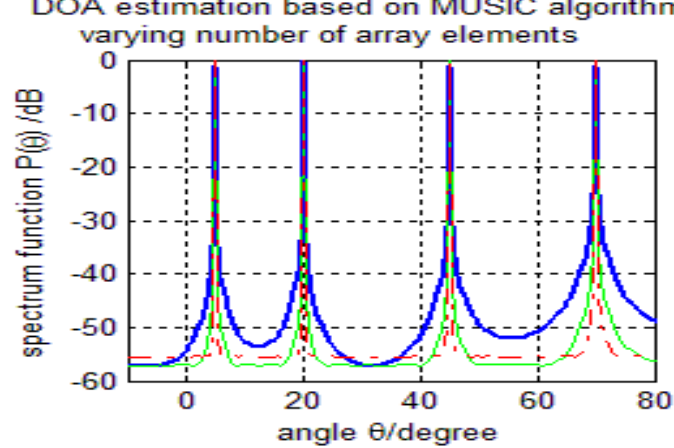

(b)

Fig 4: (a) DOA of four signals using MUSIC algorithm (b) DOA estimation by varying number of array elements

These signals are recognized by the MUSIC algorithm. As seen from Fig. 3, the various peaks shown with blue colour represents the four signals with angle of incidence as $5^{\circ}, 20^{\circ}, 45^{\circ}, 70^{\circ}$ estimated using MUSIC algorithm. The number of incident signals, their direction of arrival is seen with sharp peaks and high precision. Varying the number of array elements as 10,30 and 90 in above, simulation is done and results are obtained. As seen from Fig. 4(a), blue line, green line and red line represents the number of array elements as 10, 30 and 90 respectively. As the number of array elements are increased, more accurate DOA estimates has been achieved. Also, the spectral bandwidth becomes narrow. But the only disadvantage is that it requires more processing power. So it has to be selected wisely without wasting the resources.

Simulation in Fig. 4(b) shows the relationship between Direction of Arrival and spacing between the array elements. Spacing between the array elements are varied as $\lambda / 5, \lambda / 2$, $\lambda$. As can be seen from the figure that green line, blue line and red line represents the spacing between array elements as $\lambda / 5, \lambda / 2$ and $\lambda$ respectively. When the array spacing is less than $\lambda / 2$, then MUSIC algorithm gives a good accuracy. But when the spacing is more than $\lambda / 2$, the spectrum starts showing false peaks and loses its spectrum accuracy. Thus the element spacing should not be kept more than $\lambda / 2$. 


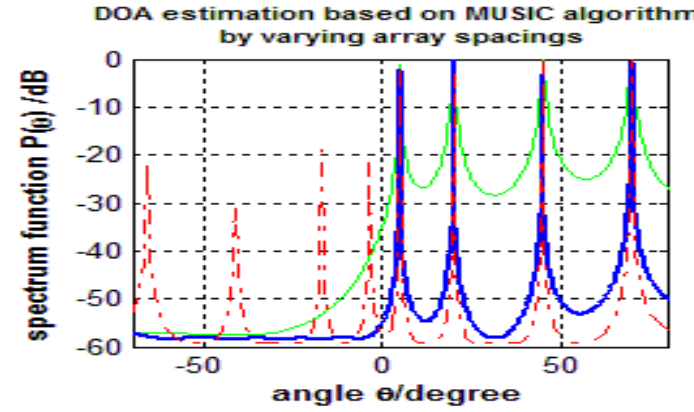

(a)

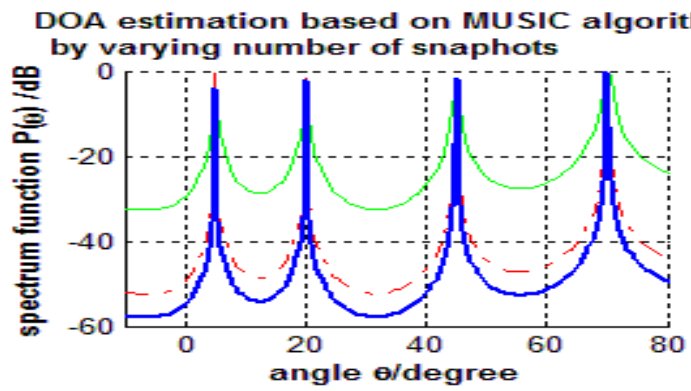

(b)

Fig. 5: (a) DOA estimation by varying array spacing (b) DOA estimation by varying number of snapshots

Simulation in Fig. 5(a) and 5(b) shows the relationship between Direction of Arrival estimation and number of snapshots. Number of snapshots is varied as 10, 40 and 200 and simulation was done. The results are shown in Fig. 5(a) and 5(b). As seen, red line, green line and blue line represents the number of snapshots as 10, 40 and 200 respectively. With the increase in number of snapshots, DOA estimation accuracy increases, but at the same time computational load increases. So it has to be selected wisely.

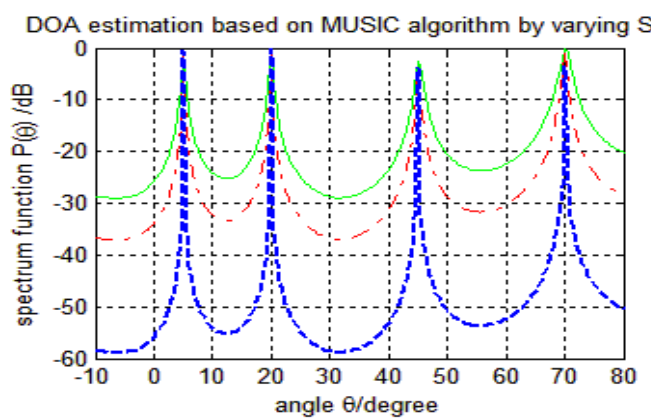

(a)

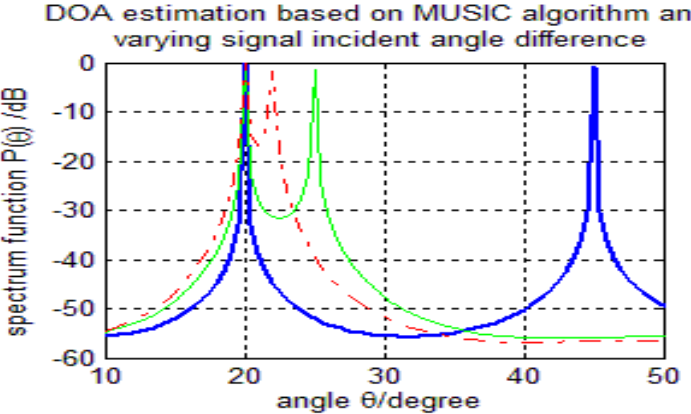

(b)

Fig. 6: (a) DOA estimation by varying SNR (b) relationship between DOA estimation and incident angle difference
Fig. 6(a) shows the simulation results of DOA estimation by varying SNR. Green, Red and Blue lines represent SNR at $10 \mathrm{~dB}, 0 \mathrm{~dB}$ and $20 \mathrm{~dB}$ respectively. At high SNR the performance of DOA estimation is more accurate and is represented by sharp peaks compared to low SNR. Thus the value of SNR also affects the performance of estimation algorithms. Fig. 6(b) shows the relationship between DOA estimation and incident angle difference. Incident angles are taken as $20^{\circ}, 22^{0} ; 20^{\circ}, 25^{\circ} ; 20^{\circ}, 45^{\circ}$ which are shown with red, green and blue colours respectively. On increasing the angle difference, the beam-width of the spectrum becomes narrow. Also, MUSIC algorithm is able to provide resolution much better than $2^{\circ}$.

\section{CONCLUSION}

DOA estimation has wide range of applications in the field of radar, communications, seismic survey and biomedicine etc. In this paper, a mathematical model of DOA estimation along with various algorithms is provided. Further MUSIC algorithm is simulated in detail by varying its various parameters using MATLAB. In a variety of DOA estimations which are based on spatial spectrum estimation, the MUSIC method has a higher resolution, a moderate amount of computation and better robustness in array structure. Here detailed algorithm analysis and performance evaluation for subspace based multiple signal classification (MUSIC) algorithm has been studied. In the light of above results it is evident that subspace based high resolution multi-channel MUSIC algorithm is statistically proficient and is a promising method for utilized against military communication in an electronic warfare direction finding systems.

\section{REFERENCES}

[1] HongY Wang, Modern Spectrum Estimation, Dongnan University Press, 1990.

[2] Eder, Rafael, and Johannes Gerstmayr. "Special Genetic Identification Algorithm with smoothing in the frequency domain." Advances in Engineering Software 70 (2014): 113-122.

[3] Yaakov, B., and Li, X., Estimation and Tracking: Principle, Techniques, Artech House, 1995.

[4] Xianda Zhang, Zheng Bao, Communication Signal Processing, National Defence Industry Press, 2000.

[5] Xianda Zhang, Modern Signal Processing, Tsinghua Press, 2000.

[6] Haykin,S. (1985), Radar array processing for angle of arrival estimation, in: S, Haykin, ed., Array signal processing, Prentice -Hall, Englewood Cliffs, NJ, ch, 4, 194-292.

[7] C.H Yu, J.L Li. A White Noise Filtering Method for DOA Estimation of Coherent Signals in Low SNR. Signal Processing. July 2012. Vol. 28. No. 7. pp 957-62.

[8] L.N Yang. Study of Factors Affecting Accuracy of DOA. Modern Rader. June 2007. Vol. 29. No. 6. pp 70-3.

[9] Zhang XF, Chen C, Li JF, Xu DZ. Blind DOA and Polarization Estimation for Polarization-sensitive Array using Dimension Reduction MUSIC. Multidimensional Systems and Signal Processing. Jan, 2014, 25-1. P67 82.

[10] Peter Stonica etc. MUSIC Maximum Likelihood and Cramer-Rao Bond, IEEE Trans on ASSP. 1989. Vol. 37(5). P720 741. 
[11] Harry B. Lee etc. Resolution Threshold Beamspace MUSIC for Two Closely Spaced Emitters. IEEE Trans on ASSP. 1990. Vol. 38(9). P723 738.

[12] Fei Wen, Qun Wan, Rong Fan, Hewen Wei. Improved MUSIC Algorithm for Multiple Noncoherent Subarrays. IEEE Signal Processing Letters. Vol. 21, no. 5, May, 2014.

[13] Greensted, “Delay sum beamforming." [Online]. Available:

http://www.labbookpages.co.uk/audio/beamforming/dela ySum.html
[14] Demba E. Ba, Dinei Florencio and Cha Zhang, "Enhanced MVDR Beamforming for Arrays of Directional Microphones," in proceedings ICME, IEEE, July 2007.

[15] Christ D. Richmond, "Adaptive Array Detection, Estimation and Beamforming," Workshop on Stochastic Eigen-Analysis and its Applications, MIT Lincoln Laboratory, July 10th 2006.

[16] Gilat Amos. MATLAB: an Introduction with Application. 3rd edition. Wiley; John Wiley. Cop 2008. 